EPJ Web of Conferences 71, 00106 (2014)

DOI: 10.1051/epjconf/20147100106

(C) Owned by the authors, published by EDP Sciences, 2014

\title{
Upgrade of the ALICE Experiment
}

\author{
Thomas Peitzmann ${ }^{1, a}$ for the ALICE collaboration \\ ${ }^{1}$ Nikhef and Utrecht University, The Netherlands
}

\begin{abstract}
While groundbreaking measurements on the properties of strongly interacting matter in $\mathrm{p}+\mathrm{p}, \mathrm{p}+\mathrm{A}$ and $\mathrm{A}+\mathrm{A}$ collisions at the LHC are being performed, it is clear that many important questions in heavy-ion physics will remain unanswered in this first phase of beam times up to 2017. The ALICE collaboration is setting up a program of detector upgrades to be installed in the LHC shutdown planned for $\approx 2018$, to address the new scientific challenges. We will discuss examples of the scientific frontiers and upgrade projects under study for the ALICE experiment.
\end{abstract}

\section{Introduction: the physics frontiers for ALICE}

In the first years of operation ALICE has demonstrated its excellent capabilities to measure highenergy nuclear collisions at the LHC, delivering exciting results on elliptic flow, which appears to be even larger than at RHIC [1], strong quenching of high momentum hadrons including first measurements using identified open charm mesons [2] and intriguing results on the centrality dependence of charmonium production [3], to just name a few examples. Further data taking up to 2017 will improve the understanding of heavy-ion collisions significantly and will very likely yield comprehensive results on many experimental probes, as e.g. the details of anisotropic flow of light hadrons, inclusive momentum spectra of heavy-flavour mesons including their nuclear modification, or the global features of jets in nuclear collisions, all-in-all the baseline program of the ALICE experiment.

Still, heavy-ion collisions at the LHC have the potential for important further progress in the field, with heavy-flavour measurements taking over the role of light-hadron observables from the existing measurements. Some of those very interesting light-hadron observables have turned out to be of limited sensitivity, e.g. jet quenching observed in light-hadron measurements shows an almost maximal suppression, as seen in the small values of the nuclear modification factor $R_{\mathrm{AA}}$ of unidentified charged hadrons as displayed in Fig. 1. Thus small variations in the medium parameters (e.g. colourcharge density) will have little effect on these observations. Heavy quarks are expected to loose less energy, which should make corresponding measurements more sensitive to the medium properties. First measurements of $R_{\mathrm{AA}}$ of D mesons show indications of different behaviour consistent with these expectations (see Fig. 1), albeit with large statistical and systematic errors. Measurements of this, which are precise enough to effectively constrain models, or other, even more selective measurements will not be possible.

For illustration let us consider the study of collective motion of charm. This is again a more sensitive probe than the same measurement of light hadrons, as heavy quarks (or hadrons) should be

ae-mail: t.peitzmann@uu.nl 


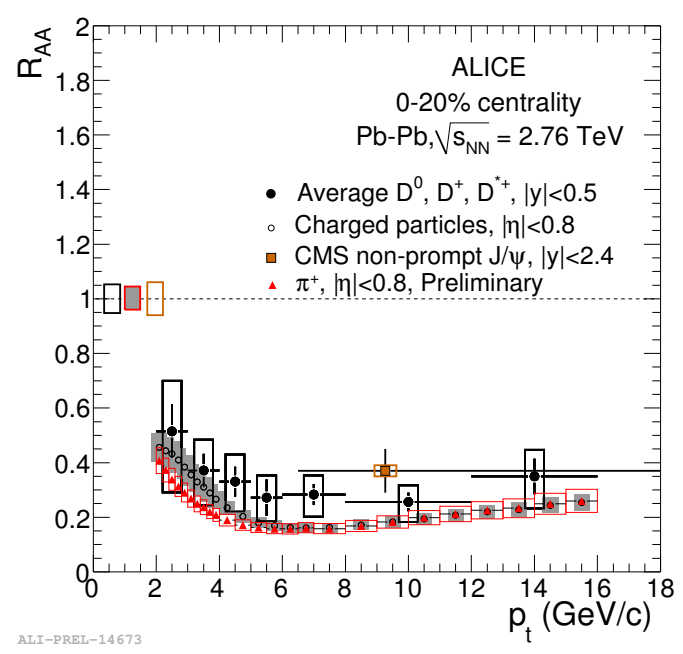

Figure 1. Average $R_{\mathrm{AA}}$ of $\mathrm{D}$ mesons as a function of $p_{\mathrm{T}}$ for the $0-20 \%$ most central $\mathrm{Pb}-\mathrm{Pb}$ collisions. For comparison also $R_{\mathrm{AA}}$ of charged hadrons is shown, as well as $R_{\mathrm{AA}}$ of non-prompt $J / \psi$ as measured by CMS [4].

more difficult to thermalise. While the measurement of spectra of open charm mesons is challenging, it has already been shown to be feasible, and a further increase in statistics to $0.1 \mathrm{nb}^{-1}$ (or $1 \mathrm{nb}^{-1}$ for rare triggered probes) should allow measurements of the most abundant D mesons. However, anisotropic flow studies generally require an order of magnitude higher statistics, thus calling for $\approx 10 \mathrm{nb}^{-1}$ in $\mathrm{Pb}-\mathrm{Pb}$ collisions. In addition, it is known from light hadrons, that significant differences essential for theoretical understanding of collective flow are observed between mesons and baryons. This requires a measurement of charmed baryons, as e.g. the $\Lambda_{c}$. Furthermore, it is crucial for studies of equilibration properties to cover the lowest transverse momenta possible. Both, the measurement of the $\Lambda_{c}$, and a significant measurement at very low $p_{T}$ will profit enormously from an improved background rejection and secondary vertex resolution of the tracking detectors. Similar arguments hold for other important physics signals of the quark-gluon plasma, that will not be accessible with the current apparatus and the available integrated luminosity up to the long shutdown in 2017/18. Overall, an upgraded ALICE setup at high luminosity should allow

1. precision studies of primary charm (open and hidden) including flow and correlations,

2. measurements of low mass lepton pairs and thermal photons,

3. studies of jet modification via gamma-jet and jet-jet correlations including particle ID, and

4. the search for heavy nuclear states.

ALICE is preparing an upgrade program that builds on the existing strengths of the experimental setup, such as excellent tracking performance, in particular at low momenta, efficient secondary vertex reconstruction, very low material budget, and excellent particle identification. The upgraded ALICE detector will significantly improve the performance in most of these areas, except for particle identification, which will be preserved. Furthermore, the upgraded experimental setup will have enhanced 
Table 1. Summary of the physics reach: minimum accessible $p_{\mathrm{T}}$ and relative statistical uncertainty in $\mathrm{Pb}-\mathrm{Pb}$ collisions for an integrated luminosity of $\left(10 \mathrm{nb}^{-1}\right.$. For heavy flavour, the statistical uncertainties are given at $p_{\mathrm{T}}=2 \mathrm{GeV} / c$. For elliptic flow measurements, the value of $v_{2}$ used to calculate the relative statistical uncertainty $\sigma_{v_{2}} / v_{2}$ is given in parenthesis.

\begin{tabular}{lcc}
\hline Observable & $p_{\mathrm{T}}^{\min }(\mathrm{GeV} / c)$ & statistical uncertainty \\
\hline & Heavy Flavour & $($ at $2 \mathrm{GeV} / c)$ \\
\hline $\mathrm{D}$ meson $R_{\mathrm{AA}}$ & 0 & $0.3 \%$ \\
$\mathrm{D}_{\mathrm{s}}$ meson $R_{\mathrm{AA}}$ & $<2$ & $3 \%$ \\
$\mathrm{D}$ meson from B decays $R_{\mathrm{AA}}$ & 2 & $2 \%$ \\
$\mathrm{~J} / \psi$ from B $R_{\mathrm{AA}}$ & 1 & $5 \%$ \\
$\mathrm{~B}^{+}$yield & 3 & $10 \%(>3 \mathrm{GeV} / c)$ \\
$\Lambda_{\mathrm{c}} R_{\mathrm{AA}}$ & 2 & $15 \%$ \\
Charm baryon-to-meson ratio & 2 & $15 \%$ \\
$\Lambda_{\mathrm{b}}$ yield & 7 & $20 \%(7-10 \mathrm{GeV} / c)$ \\
$\mathrm{D}$ meson elliptic flow $\left(v_{2}=0.2\right)$ & 0 & $3 \%$ \\
$\mathrm{D}_{\mathrm{s}}$ meson elliptic flow $\left(v_{2}=0.2\right)$ & $<2$ & $8 \%$ \\
$\mathrm{D}$ from B elliptic flow $\left(v_{2}=0.1\right)$ & 2 & $20 \%$ \\
$\mathrm{~J} / \psi$ from B elliptic flow $\left(v_{2}=0.1\right)$ & 1 & $30 \%$ \\
$\Lambda_{\mathrm{c}}$ elliptic flow $\left(v_{2}=0.15\right)$ & 3 & $20 \%(3-6 \mathrm{Gev} / c)$ \\
\hline \multicolumn{2}{c}{ Dielectrons } \\
\hline Temperature (intermediate mass) & \multicolumn{1}{c}{$10 \%$} \\
Elliptic flow $\left(v_{2}=0.1\right)$ & 0.3 & $10 \%$ \\
Low-mass spectral function & Hypernuclei \\
\hline & $20 \%$ \\
\hline${ }_{\Lambda}^{3} \mathrm{H}$ yield & $2 \%$ \\
\hline
\end{tabular}

Table 2. Possible running scenario for the operation of the upgraded ALICE detector.

\begin{tabular}{llll}
\hline year & beams & int. luminosity & remarks \\
\hline 2019 & $\mathrm{~Pb}-\mathrm{Pb}$ & $2.85 \mathrm{nb}^{-1}$ & \\
2020 & $\mathrm{~Pb}-\mathrm{Pb}$ & $2.85 \mathrm{nb}^{-1}$ & low magnetic field \\
2021 & $\mathrm{pp}$ & t.b.c. & reference run \\
2022 & - & - & long shutdown 3 \\
2023 & - & - & long shutdown 3 \\
2024 & $\mathrm{~Pb}-\mathrm{Pb}$ & $2.85 \mathrm{nb}^{-1}$ & \\
2025 & $\mathrm{~Pb}-\mathrm{Pb}$ & $1.42 \mathrm{nb}^{-1}$ & $50 \%$ of beamtime \\
& $\mathrm{p}-\mathrm{Pb}$ & $50 \mathrm{nb}^{-1}$ & $50 \%$ of beamtime \\
2026 & $\mathrm{~Pb}-\mathrm{Pb}$ & $2.85 \mathrm{nb}^{-1}$ & \\
\hline
\end{tabular}

rate capabilities to allow an inspection of $\mathrm{Pb}-\mathrm{Pb}$ collisions at an interaction rate of $50 \mathrm{kHz}$. This is necessary to be able to exploit the physics potential, because many of the signals have an irreducibly low signal-to-background ratio requiring a high statistics measurement, but at the same time making them untriggerable. Fortunately, the signal-to-background for these observables will be much better in pp, such that only a moderately higher luminosity $(\approx 200 \mathrm{kHz})$ will be needed for reference measurements. At appropriate luminosity the upgraded apparatus will allow us to collect sufficient reference statistics in short reference runs. Crucial parts of the upgrade are the development and implementation 
of a significantly improved inner tracking system (ITS), the replacement of the TPC readout chambers with GEMs, a general upgrade of the trigger detectors (e.g. V0, T0) and of all readout electronics, and a new system integrating DAQ, High-Level Trigger and offline computing. Examples for the expected performance for important observables are given in Table 1. More information on the improvements and further details on the ALICE upgrade can be found in [5].

Another new detector, the Muon Forward Tracker, will be implemented to further enhance the measurement capabilities for the signals mentioned above via the improved forward measurement of quarkonia and low-mass dimuons, as well as heavy-flavour muons. Installation of these detector upgrades is foreseen for the LHC long shutdown 2, implying a measurement program for $\mathrm{pp}, \mathrm{p}-\mathrm{Pb}$, and $\mathrm{Pb}-\mathrm{Pb}$ extending up to at least $\approx 2026-$ a possible scenario for the measurement program is summarised in Table 2.

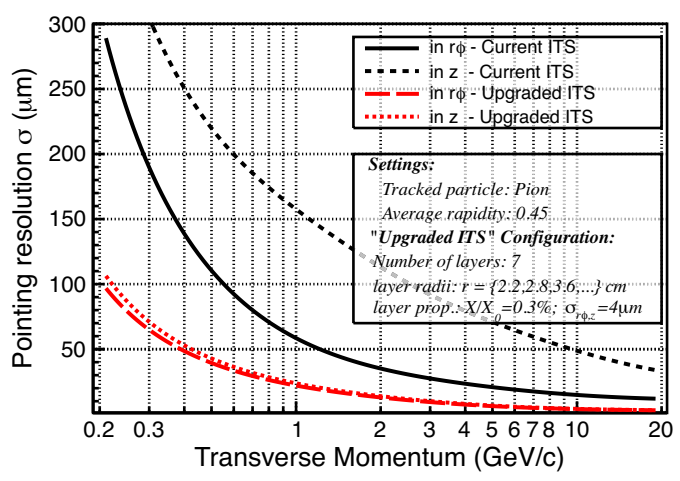

Figure 2. Track impact parameter resolution in $r \phi$ and $z$ for the current (black) and upgraded (red) ITS.

Additionally, with an appropriate detector at large rapidity an opportunity arises for measurements, where the influence of small- $x$ partons is more and more important. This is the region, where effects of gluon saturation [6] should be most prominent. Signals consistent with gluon saturation have been observed at RHIC [7], but these remain inconclusive, because the interpretation is hampered by the very limited kinematical reach. The larger beam energy of the LHC will allow us to enter a new physics regime with access to much smaller values of $x$ and a larger phase space for saturation due to the expected larger saturation scale.

\section{The ALICE Upgrade Programme}

The existing Time Projection Chamber (TPC) [8] is a unique element of the current ALICE apparatus with its continuous tracking and particle identification. However, with its long drift time it is limiting the rate capabilities. Using the multi-wire readout chambers, a gated operation (at a maximum of 3.5 $\mathrm{kHz}$ ) is necessary to limit ion feedback to the drift volume, which would otherwise lead to intolerable space-charge distortions. To be able to inspect a significantly higher interaction rate, new readout chambers based on triple-GEMs will be developed. These would be continuously read out (no gating) and should have small enough ion feedback to allow an operation at $50 \mathrm{kHz}$ interaction rate. R\&D on the GEM readout chambers is ongoing with an emphasis on achieving the projected low ion feedback and in particular on demonstrating the necessary stability in a high multiplicity/high rate environment. In a pipelined readout data would be shipped from the TPC front-end at $10 \mathrm{MHz}$ for later inspection 
in a high level trigger system (HLT). The readout electronics of all existing detector systems will be modified to accommodate the rate capabilities. Some systems will likely share the pipelined electronics with the TPC, other detectors, e.g. the electromagnetic calorimeter (EMCal), might preferably be readout on $\mathrm{L} 1$ triggers provided by a hardware trigger unit.

The high rate upgrade scenario results in $\approx 1 \mathrm{~TB} / \mathrm{s}$ of input to the online systems, where most of the data originates from the TPC readout. This necessarily will be reduced in particular for the TPC information via cluster finding and cluster-track association, as well as standard Huffman encoding. An average output rate to tape storage of the order of $10 \mathrm{~GB} / \mathrm{s}$ after this data compression should be achievable.

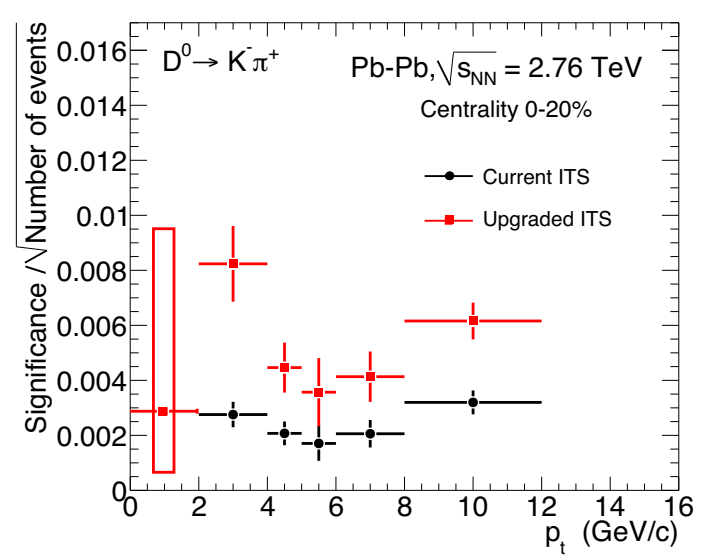

Figure 3. Significance for the measurement of the $D^{0}$ as a function of $p_{T}$ normalised to the square root of the number of events. The performance of the upgraded ITS is compared to that of the current detector.

A completely new Inner Tracking System (ITS) will be developed. The most important features of the new design in addition to enhanced rate capabilities will be:

- A decrease of the radial distance of the first layer to the beam to $22 \mathrm{~mm}$ (from $39 \mathrm{~mm}$ ),

- a reduction of the layer thickness possibly down to $50 \mu \mathrm{m}$ (from $350 \mu \mathrm{m}$ ), and

- a higher granularity with pixel sizes as small as 20x20 $\mu \mathrm{m}^{2}$.

This should be achievable by implementing seven layers of Monolithic Active Pixel Sensors and further optimisation of cabling, mechanical and cooling structures.

The new ITS will improve the secondary vertex resolution by a factor of $\approx 3$ (see Fig. 2) and allow measurements down to much lower values of $p_{T}$. Furthermore the high efficiency and low contamination of the new ITS should allow for enhanced level 2 trigger capabilities. It should give access to the measurement of charmed and beauty baryons and to the discrimination of prompt and secondary heavy quark production.

Fig. 3 shows a comparison of the expected significance for a $D^{0}$ measurement with the upgraded ITS to that expected with the current detector - in particular at low $p_{T}$ the performance improves significantly. Even more crucial is the upgrade for a measurement of the $\Lambda_{c}$ baryon in heavy-ion collisions, which cannot be measured with the current apparatus. In Fig. 4 one can see the expected statistical uncertainty for a measurement of the $\Lambda_{c} / D^{0}$ ratio in central $\mathrm{Pb}-\mathrm{Pb}$ collisions with the upgrade. 


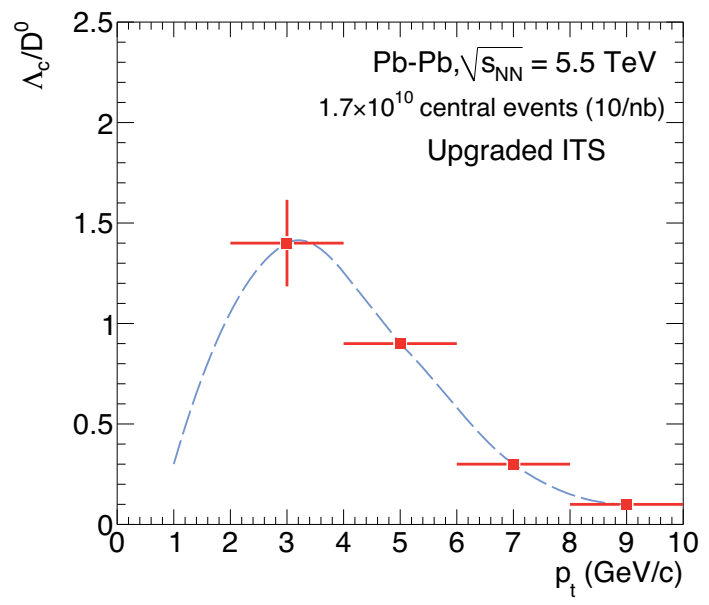

Figure 4. Possible measurement with the upgrade of the $\Lambda_{c} / D^{0}$ ratio as a function of $p_{T}$ for central $\mathrm{Pb}-\mathrm{Pb}$ collisions. The points are drawn on a line that captures the behaviour of the measured $\Lambda / K_{S}^{0}$ ratio.
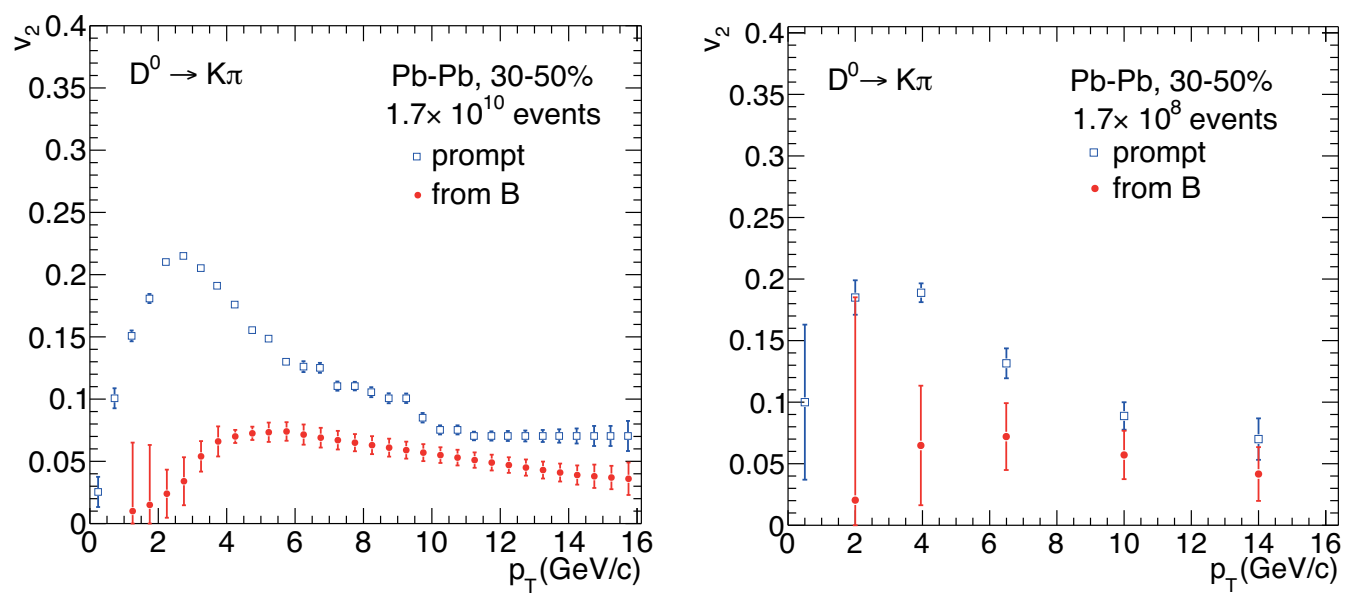

Figure 5. Estimated statistical uncertainties on $v_{2}$ of prompt and secondary $\mathrm{D}^{0}$ mesons for $1.7 \cdot 10^{10}$ events (left) in the $30-50 \%$ centrality class, which correspond to $10 \mathrm{nb}^{-1}$, and for $1.7 \cdot 10^{8}$ events (right), which correspond to about $0.1 \mathrm{nb}^{-1}$.

As another important example of the estimated performance Fig. 5 shows simulations of an elliptic flow measurement of prompt and secondary D mesons for $10 \mathrm{nb}^{-1}$ (left) and for $0.1 \mathrm{nb}^{-1}$ (right), the former of which would only be accessible with the upgrade.

The improved background rejection and the better identification of heavy-flavour electrons would also enable us to perform unprecedented measurements of low-mass dileptons. Fig. 6 shows mass spectra of the excess dielectrons after the subtraction of the combinatorial background and the correlated background from hadron decays (including heavy flavour). The errors on the contribution of 

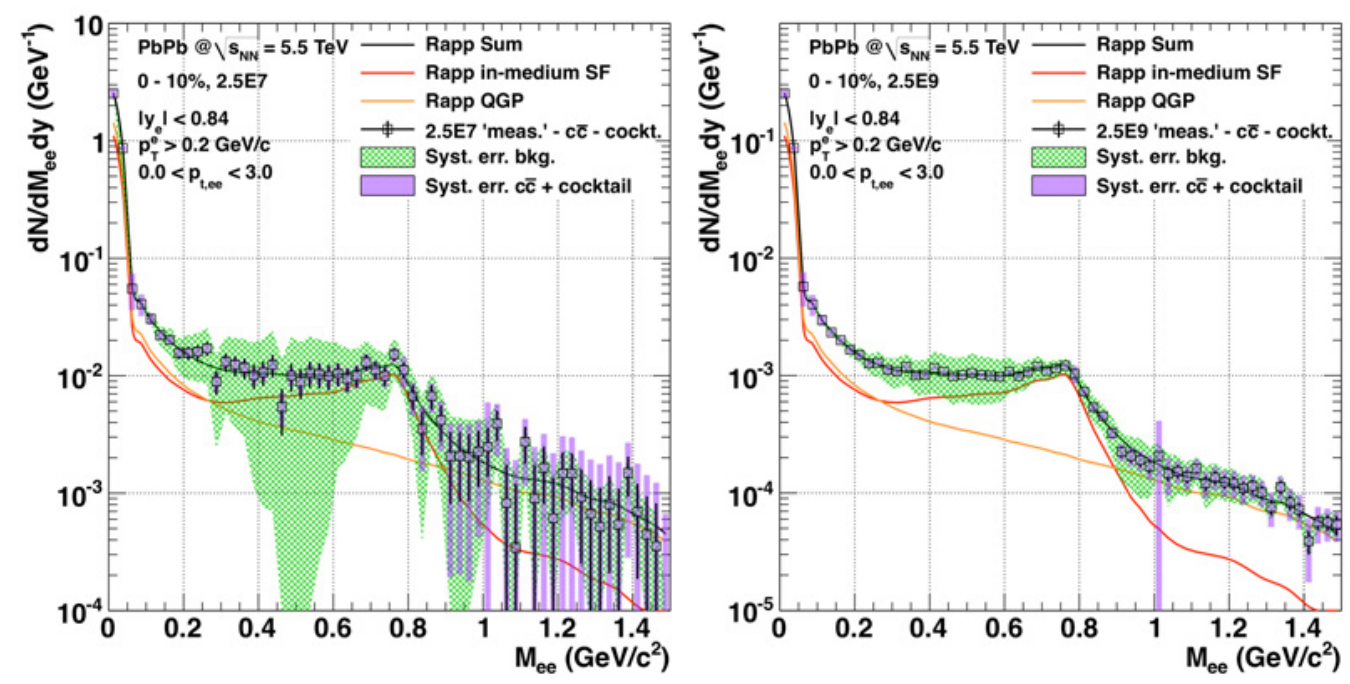

Figure 6. Invariant mass spectrum of excess $e^{+} e^{-}$pairs after the subtraction of known background sources for 0$10 \%$ most central $\mathrm{Pb}-\mathrm{Pb}$ collisions at $\sqrt{s_{N N}}=5.5 \mathrm{TeV}$. The green boxes show the systematic uncertainties from the combinatorial background subtraction, the magenta boxes indicate systematic errors related to the subtraction of the cocktail and charm contribution. The left panel shows estimates using the performance of the current ITS, the right panel the results for the upgraded ITS.

these background sources dominates the measurement without the upgrade (left panel). These systematic errors would be significantly reduced with the upgraded ITS (right panel). In addition, the comparison of the two panels demonstrates the improvement in statistical errors from the enhanced rate capabilities. These measurements would profit further from a dedicated run at lower magnetic field to improve the detector acceptance at low mass and low $p_{T}$.

More details on the design and the physics program and performance of the new ITS is described in [9].

The installation of the Muon Forward Tracker (MFT), a tracking system complementing the Muon Spectrometer, will improve the physics performance of the muon measurements at forward rapidities $(-4.0<\eta<-2.5)$. The MFT will be located upstream of the hadron absorber of the Muon Spectrometer, close to the new ITS, and will consist of five tracking planes built on the same monolithic active pixel technology as the new ITS. By adding tracking in front of the muon absorber, it would allow us to reconstruct possible secondary vertices of muon tracks. This would remove the largest limitation of the current forward muon measurements, enabling the identification of $J / \psi$ from $B$ decays, and would improve the resolution and signal-to-background ratio resulting in better general performance for measurements of quarkonia and low mass dileptons. The improvement in the signal-to-background ratio for charmonium states is demonstrated in Fig. 7. The left panel shows the performance without the MFT - here in particular the $\psi^{\prime}$ is not at all apparent. With the MFT (right panel), the background can be reduced dramatically, causing in particular the $\psi^{\prime}$ to be observable. More quantitatively, the signal-to-background ratio and systematic errors for the measurement of the $\psi^{\prime}$ in central $\mathrm{Pb}-\mathrm{Pb}$ collisions should improve by a factor of 3 - 10 depending on the transverse momentum. The expected improvement for the measurement of the corresponding nuclear modification factor with the MFT 

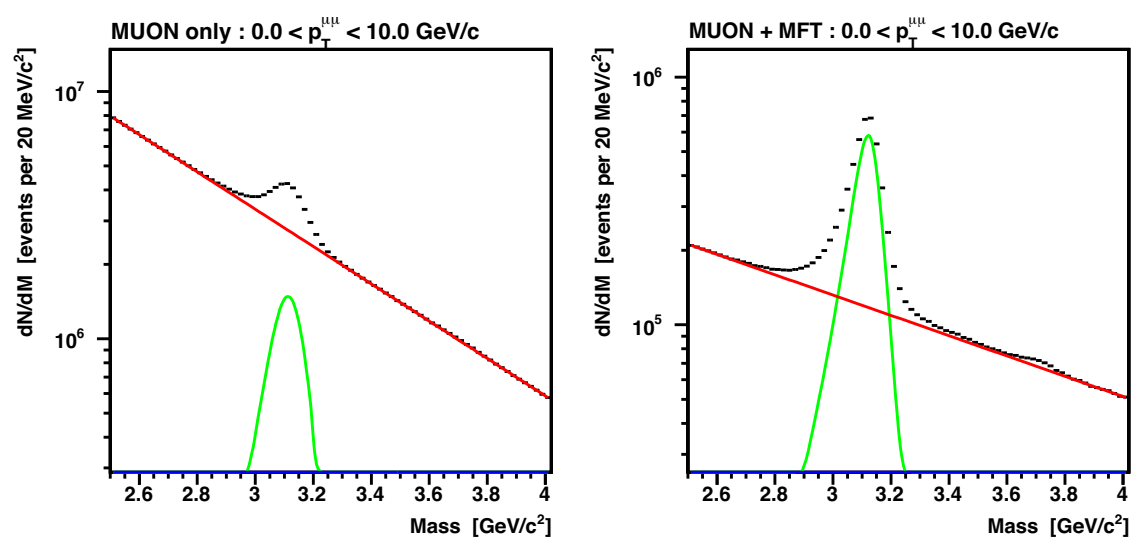

Figure 7. Mass distributions of dimuons in the vicinity of the $J / \psi$ in central $\mathrm{Pb}-\mathrm{Pb}$ collisions without MFT (left panel) and with MFT (right panel).
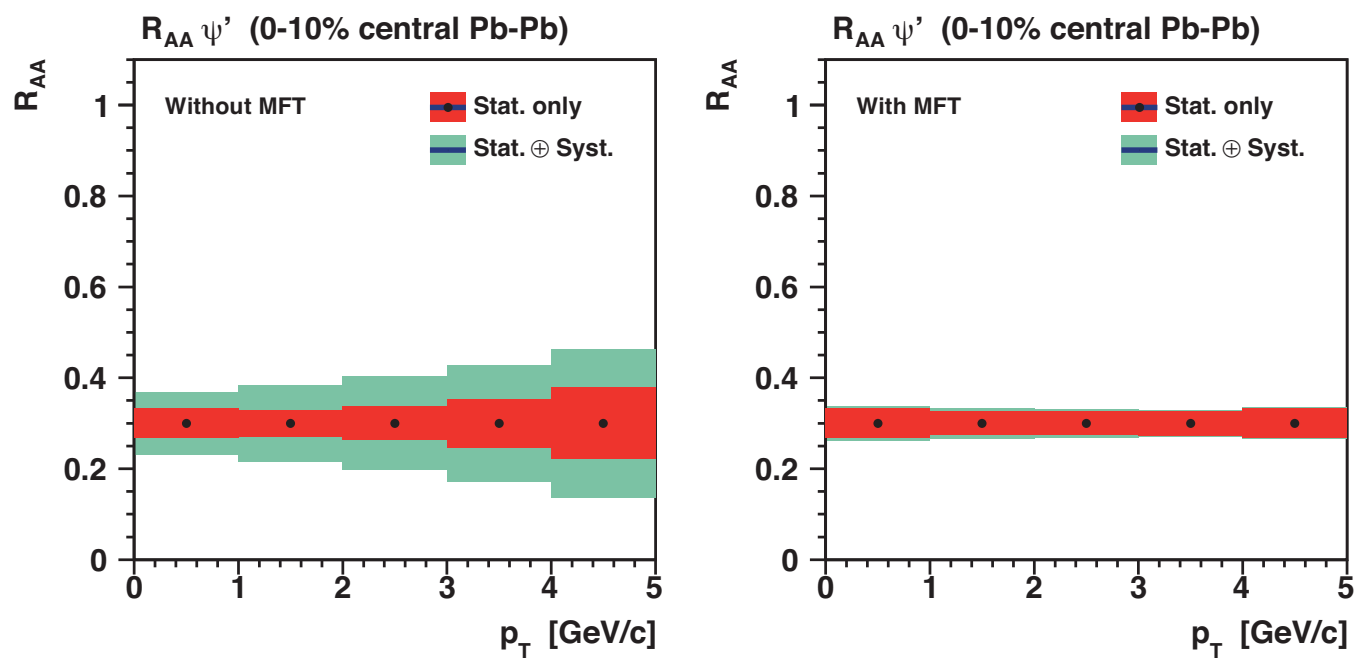

Figure 8. Expected statistical and systematical uncertainties of the nuclear modification factor $R_{A A}$ of the $\psi^{\prime}$ in central $\mathrm{Pb}-\mathrm{Pb}$ collisions without MFT (left panel) and with MFT (right panel).

compared to the performance of the existing muon arm alone is illustrated in Fig. 8. Details on the MFT performance can be found in [10].

\section{Additional Detector Upgrades}

Beyond the above upgrade programme, which has been endorsed by the LHC experiments committee (LHCC), studies are ongoing for a Forward Calorimeter (FoCal) for photon and possibly jet measurements in the forward region $(\eta \approx 3.5-5.5)$ opposite the Muon Spectrometer. The detector would 
allow a detection of neutral pions and direct photons in an $\eta$ and $p_{T}$ range not accessible to existing experiments, and would give unprecedented sensitivity to small- $x$ gluon structure and saturation effects. The $x_{2}$-range accessible with photon measurements has been estimated as shown in Fig. 9. The left panel shows the $x_{2}$ distribution for direct photon and neutral pion production at $8.8 \mathrm{TeV}$ as calculated from PYTHIA. Clearly, direct photon production is dominated $x_{2}$ values below $10^{-4}$, while pion production shows no particular low- $x$ sensitivity. While the cross section for the production of photons is significantly lower than that for pions, it is still large enough to perform measurements of high statistical precision. This is in contrast with a possible measurement of Drell-Yan pairs, which would have similar $x$-sensitivity, but extremely small cross section, which will not allow a suitable measurement in particular in $\mathrm{p}-\mathrm{Pb}$ collisions due to the limited integrated luminosity expected.

That the $x$-sensitivity is not an artefact of the simplified PYTHIA simulations can be shown in NLO pQCD calculations. While fragmentation photons can in principle obscure the access to initial state kinematic parameters, these can be suppressed significantly by an isolation cut, such that a similar $x$-sensitivity is recovered, as shown in the right panel of Fig. 9.
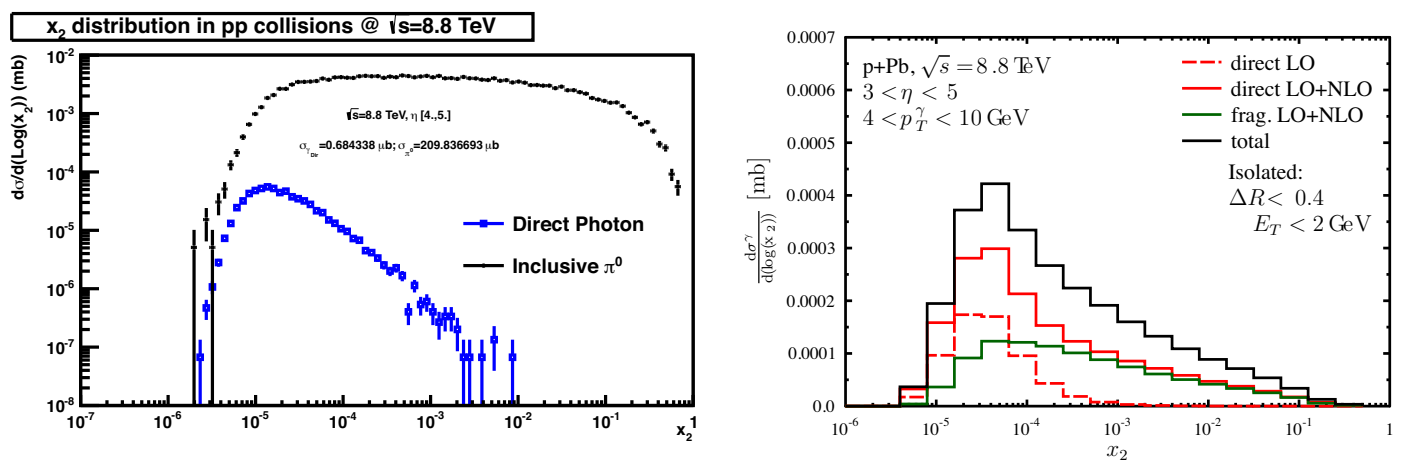

Figure 9. Distribution of $x_{2}$ values of partons contributing to direct photon production at forward rapidities in hadronic collisions at $8.8 \mathrm{TeV}$. Left: Direct photon production (blue, large symbols) in pp collisions as calculated by PYTHIA, compared to neutral pion production (black, small crosses), for $4<\eta<5$. A $p_{\mathrm{T}}$ threshold of $4 \mathrm{GeV} / c$ has been used for photons, and $2 \mathrm{GeV} / c$ for pions. Right: Isolated photons for $\Delta R<0.4, E_{\mathrm{T}}<2 \mathrm{GeV}$ from NLO pQCD calculations for $\mathrm{p}-\mathrm{Pb}$ collisions in $3<\eta<5$ using EPS09 PDFs [12].

The project is still being discussed within the collaboration, and thus installation will be considered for long shutdown 3. While the demands on energy resolution are moderate for forward measurements, the small opening angle of neutral pion decays and the overall large particle density in $\mathrm{Pb}-\mathrm{Pb}$ collisions will require good position resolution and two-particle separation power. The idea is to implement an electromagnetic calorimeter with small Molière radius and extreme granularity, most likely a SiW sandwich design with cell sizes of $\approx 1 \mathrm{~mm}^{2}$ for at least some layers, which will allow a precise direct photon measurement. For the high-granularity sensors, the same MAPS technology as for ITS and MFT is being considered. This detector would likely be complemented by a conventional hadronic calorimeter, that would improve jet reconstruction and isolation cuts for the photon measurements.

The high granularity is crucial for $\gamma / \pi^{0}$ discrimination, which is demonstrated in Fig. 10. The panel on the left hand side shows the expected performance using a detector with standard granularity $\left(1 \mathrm{~cm}^{2} \text {, corresponding to angles of } \psi \approx 0.16^{\circ}\right)^{1}$, while the right panel shows the performance

\footnotetext{
${ }^{1}$ Those conditions are in fact very similar to the ones of the electromagnetic calorimeter of the LHCb experiment.
} 

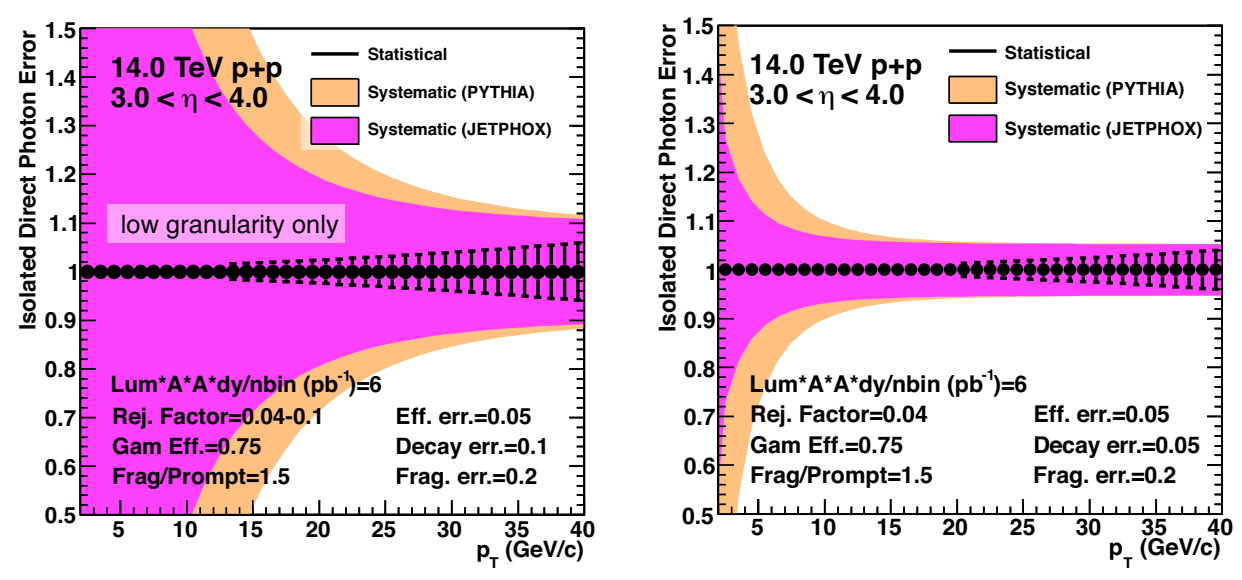

Figure 10. Estimated relative uncertainties on the cross section measurement for direct photon production in $\mathrm{p}+\mathrm{p}$ collisions at $\sqrt{s}=14 \mathrm{TeV}$, based on direct photon spectra from JETPHOX (dark band) and PYTHIA (light band), and background spectra from PYTHIA events. Statistical uncertainties are shown as error bars and the systematic uncertainty is shown as a band. This simulation assumes a location of the detector at $z=3.6 \mathrm{~m}$. Results are shown using only low granularity layers (left) and for the full detector including high granularity layers (right).

of the FoCal detector with the full granularity $\left(1 \mathrm{~mm}^{2}\right.$, corresponding to angles of $\left.\psi \approx 0.016^{\circ}\right)$. A low-granularity detector would only determine the photon yield with a much larger systematic error, mainly due to the merging of $\pi^{0}$-decay photons. Only the high-granularity option has a good sensitivity for such a photon measurement. While it would offer coverage towards higher rapidities than other LHC experiments, it is in particular the superior granularity at these large rapidities that would give FoCal a unique advantage.

\section{Summary}

ALICE has defined an upgrade program for its detector setup to enable challenging measurements in $\mathrm{Pb}-\mathrm{Pb}, \mathrm{p}-\mathrm{Pb}$ and $\mathrm{pp}$ collisions at $\mathrm{LHC}$, with at the heart a new Inner Tracking System, upgraded readout chambers for the TPC, a new forward tracking detector for muons and a general upgrade of readout electronics and $\mathrm{DAQ}$ to allow an operation with a $\mathrm{Pb}-\mathrm{Pb}$ collision rate of up to $50 \mathrm{kHz}$. The performance improvements, in particular for measurements involving open or hidden heavy flavour and low-mass dileptons, have been discussed. Only these approved upgrades will give ALICE the opportunity for unique measurements of quark-gluon plasma properties at LHC after 2018. Further upgrades for forward physics to be installed at a later stage are still being discussed in the collaboration.

\section{References}

[1] K. Aamodt et al. (ALICE), Phys. Rev. Lett. 105, 252302 (2010).

[2] B. Abelev et al. (ALICE), JHEP 09, 112 (2012). 
[3] B. Abelev et al. (ALICE), Phys. Rev. Lett. 109, 072301 (2012).

[4] S. Chatrchyan et al. (CMS), JHEP 05,063 (2012).

[5] ALICE Collaboration, Upgrade of the ALICE Experiment - Letter Of Intent, CERN-LHCC-2012012 / LHCC-I-022.

[6] L.V. Gribov, E.M. Levin, and M.G. Ryskin, Phys. Rept. 100, 1 (1983); A.H. Mueller and J.W. Qiu, Nucl. Phys. B 268, 427 (1986); L.D. McLerran and R. Venugopalan, Phys. Rev. D 50, 2225 (1994); Phys. Rev. D 49, 3352 (1994).

[7] I. Arsene et al. (BRAHMS), Phys. Rev. Lett. 93, 242303 (2004); J. Adams et al. (STAR), Phys. Rev. Lett. 97, 152302 (2006).

[8] K. Aamodt et al. (ALICE), JINST 3, S08002 (2008).

[9] ALICE Collaboration, Upgrade of the Inner Tracking System - Conceptual Design Report, CERN-LHCC-2012-013 / LHCC-P-005; Upgrade of the ALICE Inner Tracking System, CERNLHCC-2013-024 / ALICE-TDR-017.

[10] ALICE Collaboration, Addendum of the Letter Of Intent for the Upgrade of the ALICE Experiment: The Muon Forward Tracker, CERN-LHCC-2013-014 / LHCC-I-022-ADD-1.

[11] C.A. Salgado, J. Phys. G 38, 124036 (2011).

[12] I. Helenius, H. Paukkunen, K. Eskola, priv. commun. (2013). 